Journal of Applied AnALysis

Vol. 12, No. 1 (2006), pp. 83-91

\title{
UNIFORM BOUNDS FOR BESSEL FUNCTIONS
}

\author{
I. KRASIKOV
}

Received October 8, 2001 and, in revised form, July 26, 2004

\begin{abstract}
For $\nu>-1 / 2$ and $x$ real we shall establish explicit bounds for the Bessel function $J_{\nu}(x)$ which are uniform in $x$ and $\nu$. This work and the recent result of L. J. Landau [7] provide relatively sharp inequalities for all real $x$.
\end{abstract}

\section{Introduction}

Although asymptotic results for the Bessel function $J_{\nu}(x)$ have a long history, not much is known about explicit bounds. Only recently L. J. Landau [7] gave in a sense best possible upper bounds for real $x$ and $\nu \geq 0$,

$$
\left|J_{\nu}(x)\right| \leq \min \left\{b \nu^{-1 / 3}, c|x|^{-1 / 3}\right\},
$$

where $b=0.674885 \ldots$, and $c=0.7857468704 \ldots$ In fact these inequalities are sharp only in the transition region, i.e. for $x$ around $j_{\nu, 1}$, the least positive zero of $J_{\nu}(x)$. Here, using an approach developed in [3], [4], [6], we will establish pointwise inequalities for the monotonicity and the oscillatory regions, provided $\nu>-1 / 2$. Together with (1) they yield a rather precise

2000 Mathematics Subject Classification. 33C10.

Key words and phrases. Bessel function, bounds.

ISSN 1425-6908（C) Heldermann Verlag. 
set of bounds on the whole real axis. It will be convenient to introduce the parameter $\mu=(2 \nu+1)(2 \nu+3)>0$. We shall prove the following

Theorem 1. Let $\nu>-1 / 2$, then

(i) for $0 \leq x \leq \frac{\sqrt{\mu}}{2}$,

$$
\frac{J_{\nu}^{\prime}(x)}{J_{\nu}(x)} \leq \frac{4 x^{2}-12 \nu-6+\sqrt{\left(\mu-4 x^{2}\right)^{3}+\mu^{2}}}{2 x\left((2 \nu+1)(2 \nu+5)-4 x^{2}\right)} ;
$$

(ii) for $0 \leq x \leq \nu+\frac{1}{2}$,

$$
\frac{J_{\nu}^{\prime}(x)}{J_{\nu}(x)} \geq \frac{\sqrt{(2 \nu+1)^{2}-4 x^{2}}-1}{2 x} ;
$$

(iii) for $\nu+\frac{1}{2}<x<\frac{\sqrt{\mu+\mu^{2 / 3}}}{2}$, if $\nu>\frac{1}{2}$, and for $\nu+\frac{1}{2}<x<\frac{\sqrt{\mu}}{2}$ if

$$
\begin{aligned}
-\frac{1}{2}<\nu \leq & \frac{1}{2}, \\
& \frac{J_{\nu}^{\prime}(x)}{J_{\nu}(x)} \geq \frac{4 x^{2}-12 \nu-6-\sqrt{\left(\mu-4 x^{2}\right)^{3}+\mu^{2}}}{2 x\left((2 \nu+1)(2 \nu+5)-4 x^{2}\right)} .
\end{aligned}
$$

Theorem 2. Let $\nu>-1 / 2$, then for $x>\sqrt{\mu+\mu^{2 / 3}} / 2$,

$$
J_{\nu}^{2}(x) \leq \frac{4\left(4 x^{2}-(2 \nu+1)(2 \nu+5)\right)}{\pi\left(\left(4 x^{2}-\mu\right)^{3 / 2}-\mu\right)} .
$$

Moreover, the estimate is sharp in the sense that

$$
J_{\nu}^{2}(x) \geq \frac{4\left(4 x^{2}-(2 \nu+1)(2 \nu+5)\right)}{\pi\left(\left(4 x^{2}-\mu\right)^{3 / 2}+\mu\right)},
$$

at all the roots of the equation:

$$
\frac{J_{\nu}^{\prime}(x)}{J_{\nu}(x)}=\frac{\left(x^{2}-1\right) \sqrt{4 x^{2}-\mu}+x^{2}-\mu}{x\left(\sqrt{4 x^{2}-\mu}+\mu-2 x^{2}\right)},
$$

that is at a point between every two consecutive zeros of $J_{\nu}(x)$.

Comparing (2)-(4) with the well-known asymptotic formulae one can see that upper bound (2) is essentially more precise than (3), (4). Concerning Theorem 2 it is worth noticing that

$$
j_{\nu 1}>\nu+1.85576 \nu^{1 / 3}>\frac{\sqrt{\mu+\mu^{2 / 3}}}{2}
$$

for $\nu \geq 0,[9,10]$. Thus, (5) provides bounds for the envelope of $\left|J_{\nu}(x)\right|$ in the whole oscillatory region with an error of order $O\left(\nu /\left(4 x^{2}-\nu^{2}\right)\right)$ for 
sufficiently large $\nu$ and $x$. However in the transition region both theorems become very poor and should be replaced by (1). Nevertheless, since our approach is applicable in more general situations, it is worth noticing that there is a way to interpolate between the regions of Theorems 1 and 2 . We shall provide some details in the sequel.

Our main tools are the following inequalities

$$
\begin{aligned}
& U_{2}(f)=f^{2}-f f^{\prime \prime} \geq 0, \\
& U_{4}(f)=3 f^{\prime \prime 2}-4 f^{\prime} f^{\prime \prime \prime}+f f^{(4)} \geq 0,
\end{aligned}
$$

which are special cases of a more general result

$$
U_{2 m}(f(x))=\frac{1}{2} \sum_{j=0}^{2 m}(-1)^{m+j}\left(\begin{array}{c}
2 m \\
j
\end{array}\right) f^{(j)}(x) f^{(2 m-j)}(x) \geq 0,
$$

$m=0,1, \ldots$ These inequalities are valid for $f \in \mathcal{L}-\mathcal{P}$, i.e. the LaguerrePolya class consisting of real entire functions having a representation of the form

$$
c x^{n} e^{-\alpha x^{2}+\beta x} \prod_{k=1}^{\omega}\left(1+\frac{x}{x_{k}}\right) e^{-x / x_{k}} \quad(\omega \leq \infty),
$$

where $c, \beta, x_{k}$ are real, $\alpha \geq 0, n$ is a nonnegative integer and $\sum x_{k}^{-2}<\infty$, [5], [11]. The significance of this class of functions stems from the fact that it consists of these and only these functions which are uniform limits, on compact subsets of the complex plain, of polynomials with only real zeros, see e.g. [8]. The following well-known product representation

$$
J_{\nu}(x)=\frac{x^{\nu}}{2^{\nu} \Gamma(\nu+1)} \prod_{i=1}^{\infty}\left(1-\frac{x^{2}}{j_{\nu, i}^{2}}\right),
$$

where $j_{\nu, 1}<j_{\nu, 2}<\ldots$, are the positive zeros of $J_{\nu}(x)$, shows that $u=$ $x^{-\nu} J_{\nu}(x)$ is an entire function having exclusively real zeros and moreover $u \in \mathcal{L}-\mathcal{P}$. Exponential factors in the product are canceled due to the symmetry of the zeros with respect to the origin.

It is worth also noticing that expression (9) arises from the expansion

$$
|f(x+i y)|^{2}=2 \sum_{m=0}^{\infty} \frac{U_{2 m}(f(x))}{(2 m) !} y^{2 m}
$$

and thus, at least in principle, the knowledge of $U_{2 m}(f(x))$ for all $m$ would yield bounds on $|f(x)|$ in the whole complex plane. 


\section{Proofs}

Clearly, we may restrict our considerations to $x \geq 0$. In what follows we will assume $\nu>-1 / 2$, as fixed and omit it in the notation when it does not lead to confusion. We refer to [12] for all basic formulae concerning Bessel functions. It will be convenient to define the following two functions

$$
u=u(x)=x^{-\nu} J_{\nu}(x), \quad \text { and } \quad v=\frac{d u}{d x} .
$$

It can be shown directly that

$$
x u^{\prime \prime}+(2 \nu+1) u^{\prime}+x u=0 .
$$

Using (10) to eliminate $u^{\prime \prime}$ from (7) and (8), we get

$$
\begin{aligned}
U_{2}(u)= & u^{2}+\frac{2 \nu+1}{x} v u+v^{2}, \\
U_{4}(u)= & \frac{4 x^{2}-\mu}{x^{2}} u^{2}+\frac{(4 \nu+2)\left(2 x^{2}-2 \nu^{2}-5 \nu-3\right)}{x^{3}} v u \\
& +\frac{4 x^{2}-4 \nu^{2}-12 \nu-5}{x^{2}} v^{2} .
\end{aligned}
$$

It will be convenient to view both expressions as quadratics in $u$ and $v$. Notice also that the following proof yields a relatively tight bound on $j_{\nu, 1}$, namely

$$
j_{\nu, 1}>\frac{\sqrt{\mu+\mu^{2 / 3}}}{2}=\nu+0.31498 \nu^{1 / 3}+1+O\left(\nu^{-1 / 3}\right), \quad \text { if } \nu>\frac{1}{2},
$$

and

$$
j_{\nu, 1}>\frac{\sqrt{(2 \nu+1)(2 \nu+5)}}{2}, \quad \text { if }-\frac{1}{2}<\nu \leq \frac{1}{2}
$$

Proof of Theorem 1. Put $t=t(x)=v / u$. Using $2 J_{\nu}^{\prime}(x)=J_{\nu-1}(x)-$ $J_{\nu+1}(x)$, we get

$$
\frac{J_{\nu}^{\prime}(x)}{J_{\nu}(x)}=t(x)+\frac{\nu}{x}
$$

Since

$$
t^{\prime}(x)=\left(\frac{v}{u}\right)^{\prime}=-\frac{U_{2}(u)}{u^{2}} \leq 0
$$

we conclude that $t(x)$ is a decreasing function between any two consecutive zeros of $J_{\nu}(x)$. In particular, $t(x) \rightarrow-\infty$, for $x \rightarrow j_{\nu, 1}^{(-)}$. Moreover, using the power series representation of the Bessel functions,

$$
J_{\nu}(x)=x^{\nu} \sum_{i=0}^{\infty}(-1)^{i} \frac{x^{2 i}}{2^{\nu+2 i} i ! \Gamma(i+\nu+1)},
$$


one easily obtains $t(0)=0$. Thus, the graph of $t(x)$ for $x \geq 0$, consists of the decreasing branches $B_{0}, B_{1}, \ldots$, where the hyperbolic-shaped branch $B_{0}$ is defined on $\left[0, j_{\nu, 1}\right)$ changing from 0 to $-\infty$. The cotangent-shaped branches $B_{i}, i \geq 1$, are defined on $\left(j_{\nu, i}, j_{\nu, i+1}\right)$ and change from $\infty$ to $-\infty$.

To prove (3) we divide (11) by $u^{2}$, giving

$$
U_{2}(u) / u^{2}=t^{2}+\frac{2 \nu+1}{x} t+1 \geq 0 .
$$

The inequality here means that for $0 \leq x \leq \nu+1 / 2$, the function $t(x)$ lies beyond the region bounded by the roots $t_{1,2}^{*}=t_{1,2}^{*}(x)$, of the corresponding quadratic equation $t^{2}+((2 \nu+1) / x) t+1=0$,

$$
t_{1,2}^{*}(x)=-\frac{2 \nu+1 \pm \sqrt{(2 \nu+1)^{2}-4 x^{2}}}{2 x} .
$$

Comparing the graph of $t(x)$ with the graphs of $t_{1,2}^{*}(x)$, one readily finds that this is possible only if $u(x)$ has no zeros in the interval $(0, \nu+1 / 2]$, and $t(x) \geq t_{2}^{*}(x)>t_{1}^{*}(x)$. Thus, we obtain

$$
t(x) \geq-\frac{2 \nu+1-\sqrt{(2 \nu+1)^{2}-4 x^{2}}}{2 x}
$$

which is equivalent to (3).

Now we establish (2) and (4) by using similar arguments applied to (12). We shall prove the case $\nu>1 / 2$ in detail, the case $-1 / 2<\nu \leq 1 / 2$ is similar. As in the above proof of (3), the function $t(x)$ cannot intersect the solutions in $t$ of the quadratic equation

$$
\frac{4 x^{2}-4 \nu^{2}-12 \nu-5}{x^{2}} t^{2}+\frac{(4 \nu+2)\left(2 x^{2}-2 \nu^{2}-5 \nu-3\right)}{x^{3}} t+\frac{4 x^{2}-\mu}{x^{2}}=0,
$$

corresponding to (12). Multiplying this by $x^{3}$ one finds that the discriminant of the obtained quadratic is $\Delta=\left(\mu-4 x^{2}\right)^{3}+\mu^{2}$, and $\Delta \geq 0$ for $x \leq$ $\sqrt{\mu+\mu^{2 / 3}} / 2$. The solutions are $\left(t_{1}^{*}(x)\right.$ corresponds to the + sign $)$

$$
t_{1,2}^{*}(x)=\frac{(4 \nu+2)\left(2 x^{2}-(\nu+1)(2 \nu+3)\right) \pm \sqrt{\Delta}}{2 x\left((2 \nu+1)(2 \nu+5)-4 x^{2}\right)} .
$$

Let

$$
A=\frac{\sqrt{\mu}}{2}, \quad B=\frac{\sqrt{(2 \nu+1)(2 \nu+5)}}{2}, \quad C=\frac{\sqrt{\mu+\mu^{2 / 3}}}{2} .
$$

An elementary investigation of $t_{1,2}^{*}(x)$ yields that for $\nu>1 / 2, t_{2}^{*}(x)$ is a continuous function on $(0, C)$, changing from the value $-\infty$ at $x=0$ to the value

$$
t_{2}^{*}(C)=\frac{1+\mu^{1 / 3}+\sqrt{1+\mu}}{\left(2-\mu^{1 / 3}\right) \sqrt{1+\mu^{1 / 3}}}
$$


whereas $t_{1}^{*}(x)$ consists of two branches with the asymptote at $x=B$. Namely, $t_{1}^{*}(0)=t_{1}(A)=0, t_{1}^{*}(x)<0$ for $x \in(0, A), t_{1}^{*}\left(B^{-}\right)=\infty$, $t_{1}^{*}\left(B^{+}\right)=-\infty$, and $t_{1}^{*}(C)=t_{2}^{*}(C)$. A quick inspection of the graphs of $t_{1}^{*}(x), t_{2}^{*}(x)$ and $t(x)$ reveals that $t(x)$ cannot have an asymptote in $(0, C]$, since $t(0)=0$ and $t\left(j_{\nu, 1}^{-}\right)=-\infty$, and hence $j_{\nu, 1}>C$. Moreover, the only possibility is that

$$
t_{2}^{*}(x) \leq t(x) \leq t_{1}^{*}(x),
$$

where the lower bound holds on $[0, C)$ and the upper one on $[0, B)$. It can be checked that $t(x)$ is negative on $(0, C)$. Hence $t_{1}^{*}(x)$ yields only a trivial upper bound for $x>A$. Now the inequalities (13) are equivalent to (2) and (4) and the result follows.

Remark. Sharper results can be achieved on using $U_{2 m}$ for $m>2$, but they include polynomials of higher degree. For instance, $U_{4}$ gives the value of $J_{\nu}^{\prime}(\nu) / J_{\nu}(\nu)$ with an order of precision $O\left(\nu^{-1 / 2}\right)$.

To prove Theorem 2 we need the following lemma.

Lemma 1. Let $\Phi(x)=U_{4}(u) x^{2 \nu+4}$, then for $\nu>-1 / 2$ and $x>\sqrt{\mu} / 2$,

$$
\frac{\left(4 x^{2}-\mu\right)^{3 / 2}-\mu}{\pi} \leq \Phi(x) \leq \frac{\left(4 x^{2}-\mu\right)^{3 / 2}+\mu}{\pi} .
$$

Proof. First we shall prove the following claim: the function

$$
\Phi_{1}(x)=\frac{\Phi(x)}{\left(4 x^{2}-\mu\right)^{3 / 2}+\mu}
$$

increases in $x$ for $x>\sqrt{\mu} / 2$; whereas the function

$$
\Phi_{2}(x)=\frac{\Phi(x)}{\left(4 x^{2}-\mu\right)^{3 / 2}-\mu}
$$

decreases for $x>\sqrt{\mu+\mu^{2 / 3}} / 2$. To demonstrate this, define

$$
F_{i}(x)=\Phi^{\prime}(x)-z_{i} \Phi(x), \quad i=0,1,
$$

where

$$
z_{i}=\frac{6 y \sqrt{r+y^{2}}}{r\left(y^{3}+(-1)^{i}\right)}
$$

and $r=\mu^{1 / 3}, y=\sqrt{4 x^{2}-\mu} / r$. We have

$$
F_{0}(x)=\frac{3 x^{2 \nu+1}\left(r u \sqrt{r+y^{2}}+\left(r y-1+\sqrt{r^{3}+1}\right) v\right)^{2}}{y^{3}+1},
$$




$$
F_{1}(x)=-\frac{3 x^{2 \nu+1}\left(r u \sqrt{r+y^{2}}-\left(r y+1-\sqrt{r^{3}+1}\right) v\right)^{2}}{y^{3}-1} .
$$

Hence for $y \geq 0, \Phi^{\prime}(x) / \Phi(x) \geq z_{0}$, and for $y>1, \Phi^{\prime}(x) / \Phi(x) \leq z_{1}$. Integrating in $x$ the inequalities

$$
z_{0} \leq \frac{\Phi^{\prime}(x)}{\Phi(x)} \leq z_{1}
$$

and getting rid of the logarithm we get

$$
\frac{\Phi\left(x_{2}\right)}{\Phi\left(x_{1}\right)} \geq \frac{\left(4 x_{2}^{2}-\mu\right)^{3 / 2}+\mu}{\left(4 x_{1}^{2}-\mu\right)^{3 / 2}+\mu}, \quad \frac{\sqrt{\mu}}{2}<x_{1} \leq x_{2} ;
$$

and

$$
\frac{\Phi\left(x_{2}\right)}{\Phi\left(x_{1}\right)} \leq \frac{\left(4 x_{2}^{2}-\mu\right)^{3 / 2}-\mu}{\left(4 x_{1}^{2}-\mu\right)^{3 / 2}-\mu}, \quad \frac{\sqrt{\mu+\mu^{2 / 3}}}{2}<x_{1} \leq x_{2} .
$$

This justifies the claim.

Finally, using the well-known asymptotic formula

$$
J_{\nu}(x)=\sqrt{\frac{2}{\pi x}} \cos \left(x-\frac{\pi(2 \nu+1)}{4}\right)+O\left(x^{-3 / 2}\right),
$$

we obtain

$$
\lim _{x \rightarrow \infty} \frac{\Phi(x)}{x^{3}}=\frac{8}{\pi} .
$$

Now (14) follows on letting $x_{2} \rightarrow \infty$, in $\Phi_{1}(x), \Phi_{2}(x)$ and noticing that for

$$
\frac{\sqrt{\mu}}{2}<x<\frac{\sqrt{\mu+\mu^{2 / 3}}}{2}
$$

the left hand side of (14) trivially holds by $\Phi(x) \geq 0$.

Proof of Theorem 2. The proof readily follows by rewriting (12) as

$$
\begin{aligned}
& U_{4}(u)=\frac{\left(4 x^{2}-\mu\right)^{3}-\mu^{2}}{4 x^{4}\left(4 x^{2}-(2 \nu+5)(2 \nu+1)\right)} u^{2} \\
& +\frac{\left(x\left(4 x^{2}-(2 \nu+5)(2 \nu+1)\right) v+(2 \nu+1)\left(2 x^{2}-(2 \nu+3)(\nu+1)\right) u\right)^{2}}{x^{4}\left(4 x^{2}-(2 \nu+5)(2 \nu+1)\right)} \geq 0,
\end{aligned}
$$

which implies

$$
u^{2} \leq \frac{4 x^{4}\left(4 x^{2}-(2 \nu+5)(2 \nu+1)\right)}{\left(4 x^{2}-\mu\right)^{3}-\mu^{2}} U_{4}(u)
$$


for $x>\sqrt{\mu+\mu^{2 / 3}} / 2$. Combining this with the upper bound of the previous lemma which gives

$$
U_{4}(u) \leq \frac{\left(4 x^{2}-\mu\right)^{3 / 2}+\mu}{\pi x^{2 \nu+4}}
$$

we obtain (5). To establish (6) it is left to consider the case of equality in (15) and apply the lower bound of the lemma.

Since asymptotics in the transition region include the transcendental Airy function one could not expect that our approach would yield precise results in this case. But the following idea appearing in a similar context in [2] looks promising and leads to another interesting relation between $f(x)$ and $U_{2 m}(f(x))$. An explicit expression for $U_{2 m}(f(x))$ in the polynomial case, immediately implying positivity, is known [1],

$$
U_{2 m}(f(x))=f^{2}(x) \sum \frac{(2 m) !}{\left(x-x_{i_{1}}\right)^{2} \ldots\left(x-x_{i_{2 m}}\right)^{2}},
$$

where $f(x)=\left(x-x_{1}\right)\left(x-x_{2}\right) \ldots\left(x-x_{k}\right)$, and the sum is carried over all $2 m$-combinations $\left(i_{1}, \ldots, i_{2 m}\right)$ of $\{1,2, \ldots, k\}$. Let now $x_{1}$ be the least zero of $f(x)$. It is easy to see that for any polynomial $p(x)=\left(x-a_{1}\right) \ldots\left(x-a_{s}\right)$, $a_{1}<\ldots<a_{s}$, and $c<a_{1}$, we have $|p(c)|>|p(y)|$ for $c<x<2 a_{1}-c$. Applying this to the denominators of each term of (16) yields

$$
f^{2}(x)<f^{2}(c) \frac{U_{2 m}(f(x))}{U_{2 m}(f(c))},
$$

for $c<x<2 x_{1}-c$. Simple limiting arguments applied to a truncated infinite product of $J_{\nu}(x)$ show that this inequality is valid for $f(x)=u(x)$. Thus (17) enables one to extrapolate the values of $f(x)$ from the monotonicity to the transition region, provided $f(c)$ and $U_{2 m}(x)$ are known. In this connection notice that analogously to Lemma 1 one gets

$$
\frac{2 x-2 \nu-1}{\pi} \leq U_{2}(u) x^{2 \nu+2} \leq \frac{2 x+2 \nu-1}{\pi},
$$

yielding bounds for all $x$.

\section{References}

[1] Dilcher, K., Stolarsky, K. B., On a class of nonlinear differential operators acting on polynomials, J. Math. Anal. Appl. 170 (1992), 382-400.

[2] Erdelyi, T., Magnus, A. P., Nevai, P., Generalized Jacobi weights, Christoffel functions, and Jacobi polynomials, SIAM J. Math. Anal. 25 (1994), 602-614.

[3] Foster, W. H., Krasikov, I., Bounds for the extreme roots of orthogonal polynomials, Int. J. Math. Algorithms 2 (2000), 121-132.

[4] Foster, W. H., Krasikov, I., Explicit bounds for Hermite polynomials in the oscillatory region, LMS J. Comput. Math. 3 (2000), 307-314. 
[5] Jensen, J. L. W. V., Recherches sur la theorie des equations, Acta Math. 36 (1913), 181-195.

[6] Krasikov, I., Nonnegative quadratic forms and bounds on orthogonal polynomials, J. Approx. Theory 111 (2001), 31-49 .

[7] Landau, L. J., Bessel functions: monotonicity and bounds, J. Londom Math. Soc. (2) 61 (2000), 197-215.

[8] Levin, B. Ja., Distribution of Zeros of Entire Functions, Transl. Math. Monographs 5, Amer. Math. Soc., Providence, RI, 1964, revised ed. 1980.

[9] Lang, T., Wong, R., "Best possible" upper bounds for the first two positive zeros of the Bessel function $J_{\nu}(x)$ : the infinite case, J. Comput. Appl. Math. 71 (1996), 311-329.

[10] Lorch, L., Uberti, R., "Best possible" upper bounds for the first positive zeros of the Bessel function - the finite case, J. Comput. Appl. Math. 75 (1996), 249-258.

[11] Patrick, M.L., Extension of inequalities of the Laguerre and Turan type, Pacific J. Math. 44 (1973), 675-682.

[12] Watson, G. N., A Treatise on the Theory of Bessel Functions (2nd ed.), Cambridge Univ. Press, Cambridge, 1995.

\section{ILIA KRASIKov}

Department of Mathematical Sciences

BRUNEL UNIVERSITY

UXBRIDGE UB8 3PH

UNITED KINGDOM

E-MAIL: MASTIIK@BRUNEL.AC.UK 